\title{
Indicadores bióticos del cambio climático: casos granadilla y café
}

\author{
Biotic indicators of climate change: passion fruit and coffee cases
}

\author{
Juan Eduardo Gil Mora ${ }^{1, \risingdotseq}$. \\ ${ }^{1}$ Escuela de posgrado de la Universidad Andina del Cusco, Cusco, Perú. \\ ”mundoandino2005@yahoo.es
}

\begin{abstract}
Resumen
La actividad agrícola constituye un sector de interés en el país y es altamente vulnerable al cambio climático; el 73\% de la PEA rural, se dedica a esta actividad; las divisas generadas alcanzan U.S. \$ 1,800 millones. La agricultura es afectada por la escasez de agua, debido a la dependencia hídrica de nevados y humedales. El trabajo se efectuó en la Amazonía alta del Cusco, en La Convención; se evaluaron dos cultivos típicos e importantes en la economía campesina: la Granadilla (Passiflora ligularis juss) y el cafeto (Coffea arabica), que en los últimos tres decenios han sufrido consecuencias del cambio climático en las microcuencas de los ríos Saqsara y Yanatile en el distrito de Santa Teresa. Los efectos son el ataque de plagas y enfermedades, el bajo rendimiento; en razón a ello, hoy, estos cultivares se desarrollan a los 2,300 msnm, lo que antes se cosechaba a los $1,500 \mathrm{~m}$. de altitud.
\end{abstract}

Palabras claves; Cambio climático, Requerimientos ambientales, Fenología, Precipitación, Productividad.

\begin{abstract}
Agricultural activity constitutes a sector of interest in Perú; 73\% of the rural EAP is dedicated to this activity, the currencies generated reach U.S. \$ 1,800 million; it is highly vulnerable to climate change. Agriculture is affected by water scarcity, due to the water dependence of snowy and wetlands. The work was carried out in the high Amazon of Cusco, in La Convencion, two typical crops with consequences on the peasant economy were evaluated; the passion fruit (Passiflora ligularis juss) and the coffee tree (Coffea arabica) that in the last three decades have suffered consequences of climate change in the micro basins of the Saqsara and Yanatile rivers in the district of Santa Teresa; the effects are the attack of plagues and diseases, the low yield; because of this, today these cultivars are developed at 2,300 m.a.s.l., which were previously harvested at 1,500 m. of altitude.
\end{abstract}

Keywords: Climate change, Environmental requirements, Phenology, Precipitation, Productivity

Citar como: Mora, JEG. (2019). Indicadores bióticos del cambio climático: casos granadilla y café. Rev Yachay, 8(1), 522-529.

Recibido: 14-07-2019; Aceptado 12-10-2019; Publicado: 12-12-2019

\section{Introducción}

Los agricultores de la microcuenca, reportaron problemas en los cultivos de granadilla y café, los síntomas más importantes señalados son la baja producción y el ataque de plagas; aspectos que han sido materia del análisis en la investigación, junto a la evaluación de las diferencias de temperatura, precipitación y humedad relativa en la microcuenca.

La presente investigación se justifica por la relevancia social, pues los resultados no solo benefician a la comunidad científica, sino también a los agricultores; puesto que, se analizan las causas y consecuencias del cambio climático en estos dos cultivares.

El objetivo de la investigación fue contrastar la información verbal brindada por los agricultores de la sub cuenca respecto de los efectos del cambio climático en los cultivares de granadilla y café; así como, sistematizar la información primaria y secundaria obtenida en el estudio.

La hipótesis estuvo enmarcada en que cultivares, como la granadilla y el cafeto, son sensibles a los cambios de temperatura, humedad relativa y precipitación como indicadores del cambio climático y sus efectos.

Rev Yachay volumen (8) Numero (1), enero-diciembre 2019 
La investigación se justifica en razón a que, las consecuencias del cambio climático afectan sustancialmente a los menos protegidos a través de una baja producción de los cultivares, sea por el ataque de enfermedades o plagas, bajos rendimientos en la cosecha que generan pérdidas económicas y mayor esfuerzo de los agricultores; es el caso de los caficultores y granadilleros en Santa Teresa, La Convención, que durante décadas han ido perdiendo sus ingresos y ha incrementado el esfuerzo de los agricultores; hechos que impulsan a efectuar trabajos de investigación a fin de explicar los efectos e impactos del cambio climático en escenarios susceptibles de ser impactados. La granadilla y el café son indicadores importantes respecto de los impactos del cambio climático, pues son susceptibles a los cambios en temperatura, humedad relativa y precipitación; aspectos que son considerados en la presente investigación.

En el presente acápite de introducción, se describe el escenario de la investigación y que a continuación se da a conocer.

\section{Características de la microcuenca}

La cuenca del río Sacsara, se ubica en el distrito de Santa Teresa, Provincia de La Convención, Región Cusco; posee diversas altitudes que fluctúan desde 1,321 hasta los 6,158 msnm. Esta geomorfología, otorga una diversidad de pisos altitudinales y microclimas y determina que la población se dedique tanto a la agricultura tradicional, como a la actividad vinculada al mercado nacional e internacional; siendo el café y la granadilla los cultivos de mayor importancia y relevancia en ámbito de estudio (Gil et al., 1999).

\section{Características climáticas}

El clima es cálido-templado y húmedo, registrándose dos períodos: el de lluvias entre noviembre y marzo y el de secas, entre mayo y setiembre. La precipitación es de 1,500 mm anuales, con temperaturas relativamente bajas en los meses de mayo a agosto, registrándose en el piso de valle temperaturas de 10 y $11^{\circ} \mathrm{C}$. Las temperaturas más altas se observan en los meses de setiembre a diciembre, superando $\operatorname{los} 28^{\circ} \mathrm{C}$. (SENAMHI, 2013).

\section{Actividad Económica}

La agricultura es la actividad económica más importante, los cultivos más importantes son: granadilla (Passiflora ligularis juss), café (Coffea arabica), cacao (Theobroma cacao L.), plátano (Musa paradisiaca L), yuca (Manihot esculenta Crant), virraca (Arracacia xanthorrhiza), uncucha (Xanthosoma sagittifolium L. Schott), palta (Persea americana Millar), lúcuma (Pouteria lucuma), que se circunscriben a las cabeceras de la cuenca y a los suelos marginales de fuerte pendiente (Gil et al. 2009).

\section{Materiales y métodos}

Para la obtención de información y el análisis correspondiente, se utilizó la investigación participativa; con trabajos de campo, visitas guiadas y el trabajo directo con los productores y autoridades. También se utilizó información secundaria, se planificó encuestas a los productores y entrevistas con caficultores, productores de granadilla, comerciantes y autoridades locales. Igualmente, se aplicaron las observaciones de campo sobre los efectos en el crecimiento vegetativo, fenología, ataque de plagas y presencia de enfermedades.

\section{Resultados}

\section{Cultivo de la Granadilla (Passiflora ligularis juss).}

Tabla 1: Principales requerimientos ambientales para la granadilla.

\begin{tabular}{|c|c|c|c|c|}
\hline \multirow[t]{2}{*}{ Requerimientos } & \multirow{2}{*}{$\begin{array}{l}\text { Umbral mínimo } \\
\left({ }^{\circ} \mathbf{C}\right)\end{array}$} & \multicolumn{2}{|c|}{ Temperatura óptima $\left({ }^{\circ} \mathrm{C}\right)$} & \multirow[t]{2}{*}{ Umbral máximo $\left({ }^{\circ} \mathrm{C}\right)$} \\
\hline & & Mínimo & Máximo & \\
\hline Temperatura & 12 & 15 & 18 & 20 \\
\hline \multirow[t]{2}{*}{ Precipitación } & Umbral mínimo & \multicolumn{2}{|c|}{$\begin{array}{l}\text { Precipitación óptima } \\
\text { mm/año }\end{array}$} & $\begin{array}{l}\text { Umbral máximo } \\
(\mathrm{mm} / \mathrm{año})\end{array}$ \\
\hline & 1,200 & 1,500 & 2,200 & 2,500 \\
\hline \multirow[t]{3}{*}{ Humedad relativa } & \multicolumn{4}{|c|}{ Humedad relativa óptima (\%) } \\
\hline & \multicolumn{2}{|c|}{ Mínima } & \multicolumn{2}{|r|}{ Máxima } \\
\hline & \multicolumn{2}{|c|}{60} & \multicolumn{2}{|r|}{80} \\
\hline
\end{tabular}

Fuente: Adaptado de SENAMHI (2010)

Rev Yachay volumen (8) Numero (1), enero-diciembre 2019 
La granadilla constituye el cultivo con mayor rentabilidad; las plantaciones se encuentran en las microcuencas de Salkantay, Sacsara y Yanatile; en altitudes que fluctúan entre los 1,900 a 3,000 msnm. Los productores señalan que la fenología de la granadilla y café, varían según el piso ecológico donde se encuentra el cultivo. La parte baja (1,900 a 2,200 msnm), parte media (2,200 a 2,600 msnm) y parte alta (superior a 2,600 msnm); siendo esta última, la zona donde se encuentran nuevas plantaciones; estas han sido instaladas por los productores porque perciben el efecto del incremento de la temperatura en las partes bajas y medias.

\subsection{Cultivo y Problemática}

a. Piso ecológico: El cultivo se desarrolla en la parte alta aun cuando se observan plantas en plena producción en la parte media, el rendimiento es mejor entre los 1,900 y $2,300 \mathrm{msnm}$, donde las temperaturas oscilan entre 16 y $18^{\circ} \mathrm{C}$.

b. Sistema de propagación: Es mediante semillas sexuales, sin previo almacigado; las plántulas generadas de esta forma proceden de la distribución fortuita a través de la excreta de animales y humanos; consecuentemente, las plántulas no son las mejores para el trasplante. Se practica también la propagación mediante esquejes.

c. Control de plagas y enfermedades: En forma muy incipiente y con escasos resultados; siendo, en la mayoría de los casos, un control mecánico.

d. Cosecha: Se realiza cuando los frutos alcanzan la consistencia necesaria y la madurez adecuada. La primera cosecha se efectúa al año de plantación. La producción es permanente hasta el octavo año. El recojo de los frutos se efectúa en forma manual con ayuda de un implemento de fabricación casera y rústica denominada "pallana", consistente en un gancho metálico construido en base a un alambre sujetado en un palo; igualmente se suele emplear la misma base, que culmina en una bolsa de lona. La producción fluctúa entre 500 y 800 unidades de granadilla por planta y año. Existen tres épocas de cosecha. La primera, denominada por los campesinos como "mita", se inicia en marzo, la segunda en julio y la tercera en noviembre.

Efectuando un cálculo referente a la productividad resultaría entre 135 a 170 TM al año la producción de granadilla; es decir, fácilmente supera el $5 \%$ de la producción nacional en ambas microcuencas.

e. Fenología: Pueden distinguirse: reposo vegetativo, reproductiva y maduración. La fase vegetativa está formada por la sub etapa de yema vegetativa y máximo crecimiento de los brotes. La etapa reproductiva, está compuesta por las sub etapas de yema floral hinchada, botón floral, cartucho floral, flor abierta, flor fecundada, flor cuajada y, fructificación (Rivera, et al. 2002).

\subsection{Efectos del cambio climático}

a. En los meses secos (junio-agosto) la temperatura es inferior a $15^{\circ} \mathrm{C}$; la precipitación es casi nula y la humedad relativa es inferior al $50 \%$; aspectos que inciden directamente en la floración y en la aparición de plagas y enfermedades o en el marchitamiento de las plantas.

b. Cuando la precipitación es superior a 2,200 $\mathrm{mm}$ aparecen enfermedades que afectan la producción.

c. Las plantas de granadilla, cada vez se cultivan en altitudes superiores a los 2,300 msnm. Haciendo que los productores recorran más distancias y los costos de producción sean mayores en desmedro de la economía campesina.

d. Entre las plagas y enfermedades que surgen a consecuencia de las modificaciones climáticas, debido a la disminución de la precipitación y humedad relativa y, al incremento de temperatura son:

i. "Rancha", cuyo agente etiológico es Nectria haematococca y su estado anamorfo Fusarium sp. El síntoma es la clorosis o amarillamiento de las hojas.

ii. Ojo de pollo. Causado por: Phomosis sp. El daño es severo en condiciones de alta humedad. Causa clorosis generalizada de las plántulas y caída de hojas.

iii. Roña de los frutos. El agente causal es: Colletotrichum sp. Penz. Ataca el tallo principal, ramas, hojas y frutos.

iv. Entre las Plagas se tiene: larvas de lepidópteros y coleópteros que afectan a la raíz principal. Si ataca a una planta madura, provoca su muerte; igualmente se observan ácaros y nematodos (Ocampo, et al. 1993).

Rev Yachay volumen (8) Numero (1), enero-diciembre 2019 


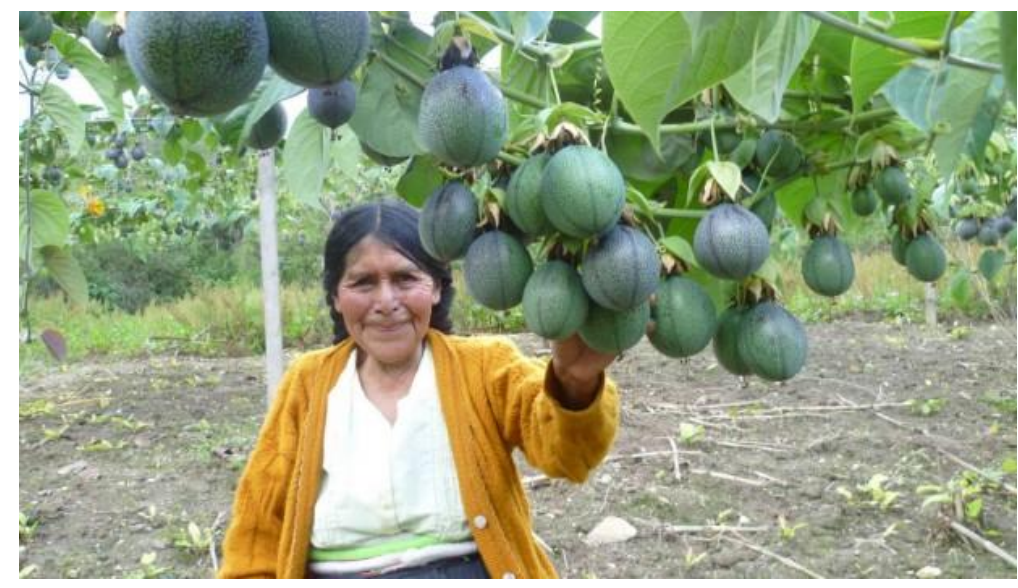

Figura 1. Mujer productora de granadilla mostrando sus frutos.

\section{Cultivo del café}

A continuación, se indican las necesidades climáticas para este cultivar.

Tabla 2: Principales requerimientos ambientales para el café.

\begin{tabular}{|c|c|c|c|c|}
\hline \multirow[t]{2}{*}{ Requerimientos } & \multirow{2}{*}{$\begin{array}{c}\text { Umbral } \\
\text { mínimo } \\
\left({ }^{\circ} \mathrm{C}\right)\end{array}$} & \multicolumn{2}{|c|}{$\begin{array}{l}\text { Temperatura } \\
\text { óptima }\left({ }^{\circ} \mathbf{C}\right)\end{array}$} & \multirow[t]{2}{*}{$\begin{array}{c}\text { Umbral } \\
\text { máximo }\left({ }^{\circ} \mathbf{C}\right)\end{array}$} \\
\hline & & Mínimo & Máximo & \\
\hline Temperatura & 10 & 18 & 22 & 30 \\
\hline \multirow[t]{2}{*}{ Precipitación } & $\begin{array}{l}\text { Umbral } \\
\text { mínimo }\end{array}$ & \multicolumn{2}{|c|}{$\begin{array}{l}\text { Precipitación óptima } \\
\text { mm/año }\end{array}$} & $\begin{array}{c}\text { Umbral } \\
\text { máximo } \\
(\mathrm{mm} / \mathrm{año})\end{array}$ \\
\hline & 1,000 & 1,400 & 1,700 & 2,000 \\
\hline \multirow{2}{*}{$\begin{array}{l}\text { Humedad } \\
\text { relativa }\end{array}$} & \multicolumn{4}{|c|}{ Humedad relativa óptima (\%) } \\
\hline & \multicolumn{2}{|c|}{ Mínima } & \multicolumn{2}{|c|}{ Máxima } \\
\hline $\begin{array}{c}\text { Plantaciones con } \\
\text { sombra }\end{array}$ & \multicolumn{2}{|c|}{70} & \multicolumn{2}{|r|}{80} \\
\hline $\begin{array}{c}\text { plantaciones sin } \\
\text { sombra }\end{array}$ & \multicolumn{2}{|c|}{50} & \multicolumn{2}{|r|}{60} \\
\hline
\end{tabular}

Fuente. Adaptado de MINAG (2003)

\subsection{Fenología del Café}

El cafeto es el cultivar permanente con mayor superficie de siembra y rentabilidad agrícola. Se encuentra distribuido en las microcuencas de Salkantay, Sacsara, Yanatile y Huadquiña; entre los 1,300 a 2,300 msnm. La distribución altitudinal donde se ubican las plantaciones, permite diferenciar tres pisos: la parte baja $(1,300$ a 1,500 msnm); parte media $(1,500$ a 1,800 msnm) y parte alta $(1,800$ a 2,300 m). Cada nivel altitudinal posee características agroclimáticas singulares haciendo que la fenología sea diferente.

La fenología del cafeto tiene cuatro etapas: reposo vegetativo, vegetativa, reproductiva y maduración. La fase de reposo vegetativo evidencia yemas vegetativas y florales, ubicadas en el tallo y ramas laterales, se encuentran en estado de latencia y normalmente coincide con el período de escasa precipitación.

\subsection{Características climáticas}

En la parte más baja de la microcuenca se registran las mayores temperaturas máximas de 28 a $32^{\circ} \mathrm{C}$; hacia las partes medias y altas, las temperaturas máximas bordean los 20 a $24^{\circ} \mathrm{C}$. En zonas de mayor altitud, las temperaturas máximas pueden variar entre 16 a $20^{\circ} \mathrm{C}$; en

Rev Yachay volumen (8) Numero (1), enero-diciembre 2019 
cambio, las temperaturas mínimas en las partes más bajas suelen alcanzar 16 a $20{ }^{\circ} \mathrm{C}$. En las zonas intermedias la temperatura mínima varía entre 11 a $16^{\circ} \mathrm{C}$, que pueden aún ser apropiadas para la producción del café. En la zona alta varían entre los 8 a $12^{\circ} \mathrm{C}$.

La estación lluviosa es de noviembre a marzo, en ocasiones se exacerba con lluvias torrenciales con periodos de hasta dos semanas consecutivas; registrándose precipitaciones de 150 a $200 \mathrm{~mm}$ en ese periodo. En la parte baja, se registran precipitaciones de 1,400 a 1,600 mm; en tanto en la parte media, la precipitación supera los 1,800 a 2,200 mm, rango de precipitación que se considera como óptima para los cultivos; en los meses secos (mayo-setiembre) la precipitación es muy escasa, con periodos totalmente secos entre mayo a agosto.

La humedad relativa alta, se registra en la época de lluvias. En cambio, en el período de secas es baja, en algunos casos menores al $50 \%$.

Tabla 3: Umbrales de temperatura para café y granadilla.

\begin{tabular}{|c|c|c|c|c|}
\hline \multirow[t]{2}{*}{ Cultivo } & \multicolumn{4}{|c|}{ Umbrales de temperatura } \\
\hline & $\begin{array}{c}\text { Rango } \\
\text { térmico } \\
\text { no apto } \\
\left({ }^{\circ} \mathbf{C}\right)\end{array}$ & $\begin{array}{c}\text { Rango } \\
\text { térmico } \\
\text { mínimo } \\
\left({ }^{\circ} \mathbf{C}\right)\end{array}$ & $\begin{array}{c}\text { Rango } \\
\text { térmico } \\
\text { óptimo } \\
\left({ }^{\circ} \mathbf{C}\right)\end{array}$ & $\begin{array}{c}\text { Rango } \\
\text { térmico } \\
\text { máximo } \\
\left({ }^{\circ} \mathrm{C}\right)\end{array}$ \\
\hline Café & $<12$ & $12-18$ & $18-22$ & $30^{>22-}$ \\
\hline Granadilla & $<12$ & $12-15$ & $15-18$ & $20^{>18-}$ \\
\hline
\end{tabular}

Fuente: Adaptado de SENAMHI. 2010

Tabla 4: Umbrales de precipitación para café y granadilla.

\begin{tabular}{lccccc}
\hline \multirow{2}{*}{ Cultivo } & \multicolumn{5}{c}{ Umbrales de temperatura } \\
\cline { 2 - 6 } & $\begin{array}{c}\text { Rango de } \\
\text { precipitación } \\
\text { no apto } \\
(\mathrm{mm})\end{array}$ & $\begin{array}{c}\text { Rango de } \\
\text { precipitación } \\
\text { mínimo } \\
(\mathrm{mm})\end{array}$ & $\begin{array}{c}\text { Rango de } \\
\text { precipitación } \\
\text { optimo } \\
(\mathrm{mm})\end{array}$ & $\begin{array}{c}\text { Rango de } \\
\text { precipitación } \\
\text { máximo } \\
(\mathrm{mm})\end{array}$ & $\begin{array}{c}\text { Rango de } \\
\text { precipitación } \\
\text { no apto } \\
(\mathrm{mm})\end{array}$ \\
\hline Café & $<1,000$ & $1,000-1,400$ & $1,400-$ & $1,700-$ & $>2,000$ \\
& & 1,700 & 2,000 & \\
\hline Granadilla & $<1,200$ & $1,200-$ & $1,500-$ & $2,200-$ & $>2,500$ \\
& & 1,500 & 2,200 & 2,500 & \\
\hline
\end{tabular}

Fuente: Adaptado de SENAMHI. 2010

Tabla 5: Umbrales de altitud y pendiente para los cultivos en estudio

\begin{tabular}{lcccccc}
\hline \multicolumn{1}{c}{ Cultivo } & \multicolumn{3}{c}{ Umbrales de altitud } & \multicolumn{3}{c}{ Umbrales pendiente del suelo } \\
\cline { 2 - 7 } & $\begin{array}{c}\text { No } \\
\text { apta } \\
(\mathbf{m s n m})\end{array}$ & $\begin{array}{c}\text { Óptima } \\
(\mathbf{m s n m})\end{array}$ & $\begin{array}{c}\text { No } \\
\text { apta } \\
(\mathbf{m s n m})\end{array}$ & $\begin{array}{c}\text { No } \\
\mathbf{a p t a} \\
(\boldsymbol{\%})\end{array}$ & $\begin{array}{c}\text { Moderadamente } \\
\text { apta }(\%)\end{array}$ & $\begin{array}{c}\text { No } \\
\text { apta } \\
(\%)\end{array}$ \\
\hline Café & $<1300$ & $\begin{array}{c}1300- \\
2300\end{array}$ & $>2500$ & $0-40$ & $40-60$ & $>60$ \\
\hline Granadilla & $<2000$ & $\begin{array}{c}2000- \\
\text { (2) }\end{array}$ & $>2700$ & $0-25$ & $25-50$ & $>50$ \\
\hline
\end{tabular}

Fuente: Adaptado de SENAMHI. 2010

El café constituye un cultivar sensible al cambio climático. Requiere temperaturas entre 18 y $22^{\circ} \mathrm{C}$; precipitaciones entre 1,400 a 1,700 $\mathrm{mm}$ anuales; de lo contrario, es afectado en la productividad.

Rev Yachay volumen (8) Numero (1), enero-diciembre 2019 


\subsection{Impactos del cambio climático}

Los caficultores indican contracciones en la producción debido a la disminución en el florecimiento, caída de la drupa e incremento de plagas y enfermedades.

El cambio climático genera impactos severos, constituyendo una amenaza para la producción, debido a los requerimientos ecofisiológicos de este cultivo como se aprecia en la figura 2.

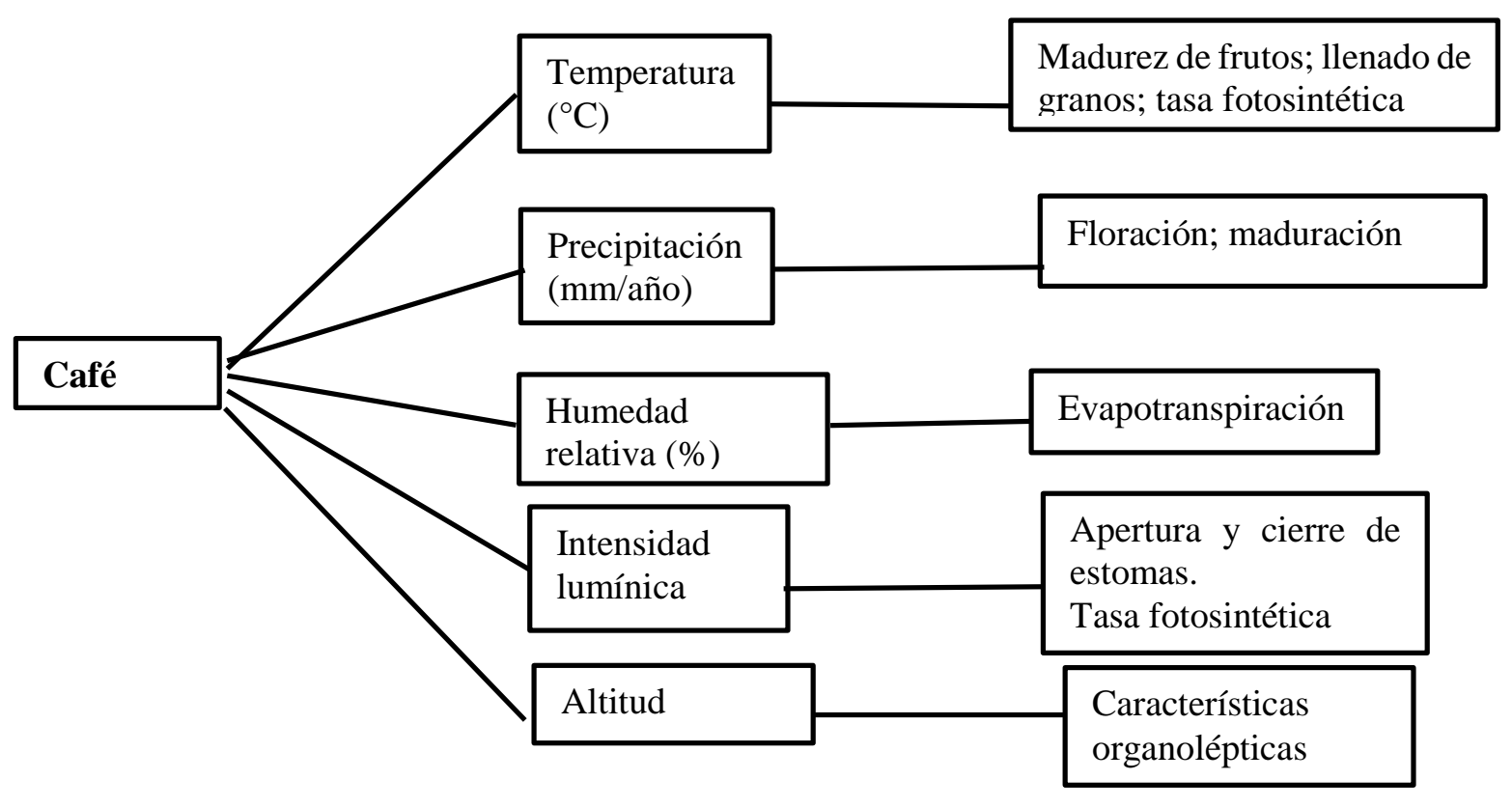

Figura 2. Factores ecofisiológicos del cultivo de café. (Fuente: Observaciones de campo.)

El cambio climático afecta restrictivamente el rendimiento y la calidad del café, los principales efectos, caracterizado por las variaciones atípicas de temperatura, humedad relativa y precipitación, se evidencian en:

a. Calidad. Cuando la temperatura se incrementa, el café madura más rápido y con inferior calidad. El café cultivado en la zona baja y media de la microcuenca, tiene un sabor y aroma inferiores comparados con el mismo café cultivado en la zona alta. De acuerdo al testimonio de los caficultores, comerciantes y consumidores, prefieren el "café de altura". Consecuentemente, existe una pérdida en la calidad debido al incremento de temperatura, lo que induce a una migración hacia mayores altitudes.

b. Rendimiento. Si se presentan temperaturas muy elevadas durante los periodos importantes para la fenología como la floración o fructificación, el rendimiento es menor.

c. Plagas y enfermedades. Las temperaturas elevadas favorecen la proliferación de plagas y enfermedades y su diseminación a otras zonas donde no se observaron antes. Entre las principales enfermedades, se tiene: la antracnosis (Collectotrichum coffeanum), la mancha de hierro (Cercospora coffeícola), el ojo de gallo (Mycena citricolor) y la roya (Hemileia vastatrix), que producen caídas de hojas y dañan el tejido leñoso de las plantas.

\section{Discusión}

\section{En cuanto a la granadilla}

La granadilla tiene gran demanda en el mercado local, regional y nacional; sin embargo, es uno de los cultivos con menos asistencia técnica. A ello se suma una comercialización deficiente caracterizada por los fluctuantes precios impuestos por el intermediario. La granadilla se cultiva entre los $500 \mathrm{~m}$ a 3,000 msnm; no obstante, el ámbito donde el cultivo se adapta mejor se halla entre los 1,200 m a 2,400 msnm (López, 2006).

Rev Yachay volumen (8) Numero (1), enero-diciembre 2019 
Los requerimientos climáticos señalan que la granadilla desarrolla mejor en un rango de temperatura, entre 15 a $18^{\circ} \mathrm{C}(\mathrm{CONAFRUT}$, 1996). Temperaturas superiores a $24^{\circ} \mathrm{C}$ generan estrés hídrico y disminuye el ciclo de vida de la planta; en cambio, temperaturas menores a $12^{\circ} \mathrm{C}$ disminuyen la fecundación e incrementan los abortos florales. La precipitación pluvial se encuentra alrededor de 1,200 a 2,500 $\mathrm{mm}$ anuales, considerándose óptimo una precipitación entre 1,500 mm a 2,200 mm por año (Castro; 2003).

La humedad relativa óptima para la granadilla, fluctúa entre 60\% a 80\% (CONAFRUT, 1996; Rivera, Miranda; et Al. (2002). Si la humedad relativa es inferior al 40\%, se observa marchitez de flores, disminución de la fotosíntesis y muerte de brotes tiernos.

\section{En lo referente al café}

En el Perú, el café es el principal producto de agro exportación tradicional. La especie cultivada predominante, es la Coffea arabica L. Se cultiva entre los 400 a 2,600 msnm. (MINAG, 2003). El piso altitudinal con condiciones de producir café de buena calidad, se halla entre los 1,200 a 1,800 msnm. (Castañeda, 2007; Lara, 2005).

La temperatura óptima reportada se halla desde los 18 y $22^{\circ} \mathrm{C}$ con extremos de $13^{\circ} \mathrm{C}$ a $24^{\circ} \mathrm{C}$ (MINAG, 2003). Se reporta que temperaturas mayores a $24^{\circ} \mathrm{C}$ aceleran el crecimiento vegetativo, intensifican la muerte de brotes y reducen significativamente la floración y fructificación; en cambio, temperaturas por debajo de $17^{\circ} \mathrm{C}$ afecta el crecimiento de las plantas (Alvarado y Rojas, 1994).

La precipitación requerida para obtener un adecuado crecimiento y desarrollo de las plantas, está entre 1,400 a 1,700 mm anuales (IICA, 2004; MINAG, 2003). La producción disminuye cuando las precipitaciones son inferiores a 1,000 mm. Durante una sequía prolongada (que suele ocurrir entre los meses de mayo a noviembre) las plantas presentan clorosis y caída de hojas. En cambio, si las lluvias superan los 2,000 mm anuales se observa una mayor incidencia de enfermedades. Es importante que la precipitación pluvial tenga una buena distribución en el año para satisfacer los requerimientos de agua que la planta necesita en las etapas de floración, llenado de grano y cosecha. (Guzmán, 2013).

Temperaturas superiores a $24^{\circ} \mathrm{C}$ acelera el crecimiento vegetativo, intensifica la muerte de brotes y reduce la floración y fructificación (Alvarado y Rojas, 1994). En cambio, temperaturas por debajo de $13^{\circ} \mathrm{C}$ (mayo-agosto) generan baja producción debido a que son afectados los procesos de floración y llenado de frutos.

\section{Conclusiones}

Primera. El cultivo de la granadilla se halla circunscrito a terrenos marginales y de fuerte pendiente; por lo tanto, las labores culturales no se desarrollan con la frecuencia ni la atención debida, estando sujeto al ataque de plagas y enfermedades.

Segunda. La granadilla, cada vez se cultiva en altitudes superiores a los 2,300 msnm, haciendo que los productores recorran más distancias y los costos de producción sean mayores en desmedro de la economía campesina.

Tercera. Los caficultores indican contracciones en la producción debido a la disminución en el florecimiento, caída de la drupa e incremento de plagas y enfermedades: esto debido a las alteraciones de precipitación, humedad relativa y temperatura.

Cuarta. Para el cultivo de café, temperaturas mayores a $24^{\circ} \mathrm{C}$ aceleran el crecimiento vegetativo, se observa una mayor muerte de brotes, reduce la floración y fructificación; periodos fríos, con temperaturas inferiores a $13^{\circ} \mathrm{C}$ provocan disminución en la producción por afectarse la floración y llenado de frutos.

Quinta. La temperatura está más relacionada con el crecimiento de la planta del cafeto. Temperaturas bajas generan un desarrollo lento y una maduración de frutos tardía. Análogamente, temperaturas altas aceleran la senescencia de los frutos, afectan la fotosíntesis, reducen el crecimiento y la producción.

Sexta. El cambio climático tiene impactos severos, constituyendo una amenaza para la producción cafetalera, debido a los requerimientos ecofisiológicos de este cultivo.

Sétima. El cambio climático afecta negativamente tanto el rendimiento como la calidad del café; en razón a ello, los caficultores han optado por migrar a las zonas más altas para compensar el incremento de temperatura.

Rev Yachay volumen (8) Numero (1), enero-diciembre 2019 


\section{Biblipografia}

Alvarado S., Melvin; Rojas C., Gilberto. (1994). Gil Mora, J.E; Ortega Dueñas, R; Caparó Cultivo y Beneficiado del café, EUNED, San José, Costa Rica. Pag. 11-15, 109-115.

Castañeda et al. (2007). Estudio del sistema de producción de café (Coffea arabica L.) en la región Frailesca, Chiapas. México. 75 pag. Universidad Autónoma de Chiapas. Facultad de Ciencias Agronómicas.

Castro L.E. (2003). Guía básica para el Lara, L. (2005). Efectos de la altitud, sombra, establecimiento y mantenimiento del cultivo de la granadilla (Passiflora ligulares). ASOHOFRUCOL. Bogotá. 75 Pag. Convenio Pos cosecha CNP-UCR.

Cerdas, M.M. 1995. Aislamiento de patógenos en frutos de granadilla (Passiflora ligularis) y en flor. Programa de Posgrado en Protección de Cultivos, (Clínica de Diagnóstico Vegetal). San José, C.R. Universidad de Costa Rica. p. 5.

CONAFRUT. (1996). Aspectos de la producción de la granadilla, manejo en pos cosecha y comercialización. Boletín Técnico $\mathrm{N}^{\circ} 01$. CONAFRUT Perú. Lima.
Calderón, A; et al. (1999). Desarrollo sustentable en la microcuenca del río Sacsara. En Desarrollo Rural Alternativo. UNSAAC; Cusco. Pag. 155-185.

IICA. (2004). Cadena Agroindustrial del café en Nicaragua. Managua. 77 pag. Libro Resumen Conferencias. producción y fertilización sobre la calidad del café (Coffea arabica L.) producido en sistemas agroforestales de la zona cafetalera nor central de Nicaragua. Tesis Magíster Scientiae. CATIE. Escuela de Posgrado. p. 92.

López R, J.A. (2006). Las Passiflora, su potencial comercial y problemática sanitaria, Memorias XXXIII congreso de Entomología SOCOLEN, Manizales. p.161- 171

MINAG. (2010). Taller Nacional de Adaptación al Cambio Climático. Exposición de Manuel Leiva, en Adaptación al Cambio
Climático para la Competitividad Agraria. p. 30. Lima: Ministerio de Agricultura Dirección General de Competitividad Agraria.

Ocampo, L.E; Cardona, W; Yépez, F; Velilla, J. A. (1993). Manejo Integrado de las plagas $y$ enfermedades en el cultivo de la Granadilla. Publicación técnica $\mathrm{N}^{\circ} 19$. ICA. Secretaria de Antioquia, Medellín. p. 26-4.

Proyecto: Adaptación al Impacto del Retroceso Acelerado de Glaciares en los Andes Tropicales-PRAA-Perú. (2010). Caracterización y aptitud agroclimática de los cultivos de café, granadilla y palto en la subcuenca de Santa Teresa - Cusco. Lima: SENAMHI-MINAM. p. 96

Rivera B, Miranda D, et al. (2002). Manejo Integral del cultivo de la Granadilla (Passiflora ligularis Juss). Universidad de Caldas. Manizales. p. 130.

SENAMHI. (2013). Escenarios de cambio climático en el Perú al 2050. Lima. 